\title{
Early Detection of Tibial Cartilage Degradation and Cancellous Bone Loss in an Ovariectomized Rat Model
}

\author{
Yinong Wang, ${ }^{1}$ Zhiwei Liu, ${ }^{1}$ Qing Wang, ${ }^{1,2}$ Qianjin Feng, ${ }^{1,2}$ and Wufan Chen ${ }^{1,2}$ \\ ${ }^{1}$ Institute of Medical Information, School of Biomedical Engineering, Southern Medical University, Guangzhou 510515, China \\ ${ }^{2}$ Guangdong Provincial Key Laboratory of Medical Image Processing, Southern Medical University, Guangzhou 510515, China
}

Correspondence should be addressed to Qing Wang; wq8740@smu.edu.cn

Received 17 August 2016; Accepted 18 December 2016; Published 15 January 2017

Academic Editor: Magali Cucchiarini

Copyright (c) 2017 Yinong Wang et al. This is an open access article distributed under the Creative Commons Attribution License, which permits unrestricted use, distribution, and reproduction in any medium, provided the original work is properly cited.

This study aimed to investigate degradation of the articular cartilage and loss of the cancellous bone in an ovariectomized (OVX) rat model simulating early human menopausal stage. Fourteen health female Sprague-Dawley rats were randomly divided into two groups ( $n=7$ per group): an OVX group that underwent bilateral ovariectomy to create an OVX model with low estrogen levels and a sham group in which only the periovarian fatty tissue was exteriorized. All the animals were sacrificed at 3 weeks after ovariectomy. The left tibiae were harvested. The articular cartilage at medial tibial plateau (MTP) and lateral tibial plateau (LTP) was assessed with quantitative high-frequency ultrasound. The cancellous bone was evaluated with micro-CT. The results indicated that, in comparison with the sham rats, the OVX rats exhibited significant alterations in acoustic parameters of the articular cartilage but insignificant changes in microarchitectural parameters of the cancellous bone in early stage of low estrogen levels. The results of this study suggest that cartilage degradation induced by estrogen reduction was detected earlier with quantitative ultrasound than that of the cancellous bone loss in 3 wk OVX rats.

\section{Introduction}

Osteoporosis (OP) and osteoarthritis (OA) are severe progressive diseases with high prevalence in the elderly and affect both men and women. However, the incidence of OP and OA increases in menopausal women. Main consequence of $\mathrm{OP}$ is the increased risk of bone fractures due to abnormalities in amount and microarchitectural arrangement of bone tissue. $\mathrm{OA}$, on the other hand, causes pain and disability of one or many synovial joints resulted from progressive degradation of the articular cartilage with alterations in the bone and surrounding structures. These two diseases play a negative role in the longevity and health of menopausal women.

Both the bone and articular cartilage are the target tissues of estrogens. It has been found that estrogen receptors (ER) such as $\operatorname{ER} \alpha$ and $\operatorname{ER} \beta$ exist in osteoclasts, osteoblasts, and articular chondrocytes [1-3]. Estrogen deficiency exacerbates bone resorption exceeding bone formation resulting in menopausal OP [4-6]. The low estrogen level induces a faster loss of knee joint cartilage in menopausal women than in men of the same age [7]. The degradation of articular cartilage is one of indications of the early $\mathrm{OA}$ and can trigger the occurrence of menopausal OA [8].

Therefore, the menopause-related changes in estrogen levels affect generation of both bone and articular cartilage and thus induce the pathogenesis of OP and OA. Due to their high prevalence in elderly women, it could be anticipated that OP and OA coexist frequently. However, a defined relationship between OP and OA has not yet clearly delineated. There are conflicting findings. Some studies reported that OA was associated with higher bone mineral density (BMD), that is, an inverse relationship between OA and OP [9-11]. Some studies suggested a positive association between OP and OA that bone loss occurred with cartilage loss in OA patients $[12,13]$. One study interestingly found that both high and low BMD conditions could induce OA [14]. Others did not find a relation between cartilage degradation and bone loss [15].

Furthermore, the occurrence of OA and OP is found to be of site-relevance [9]. However, the clearly defined siterelationship between OA and OP is still debatable. Okano 
et al. found that end-stage hip OA induced lower BMD in the calcaneus but higher BMD in the spine and radius [16]. Lethbridge-Çejku et al. reported that knee OA patients had higher BMD in spine but not in hip [17], while Multanen et al. suggested that knee OA menopausal women had higher hip bone strength [11]. Therefore, more investigations are needed to delineate associations between cartilage degradation and bone loss at different anatomic locations.

To effectively evaluate $\mathrm{BMD}$, dual-energy X-ray absorptiometry (DXA) has been widely used $[10,11,16]$. DXA, however, provides little information of bone structure. Microcomputed tomography (micro-CT), a high resolution imaging tool, is used in quantitative assessments of osteoporotic alterations and therapeutic improvements in microarchitectural characteristics of the trabecular and cortical bone in the OP animal model [18-20]. Quantitative ultrasound, on the other hand, is used to characterize the degeneration of articular cartilage [21-23].

Therefore, the present study focused on menopausal alterations in the bone and articular cartilage in the tibia. We applied bilateral ovariectomy to create an OVX rat model with low estrogen levels and then assessed early-stage alterations in the bone and articular cartilage with micro-CT and quantitative ultrasound.

\section{Materials and Methods}

2.1. Animal Care and Experimental Protocol. Fourteen female Sprague-Dawley rats, aged 10 months old and weighting 299.1 to $383.8 \mathrm{~g}$, were used in this study. They were purchased from Guangdong Medical Laboratory Animal Center, China, and individually kept in metal cages under 12-hour light-dark cycle with standard rat diet and water ad libitum. The animals were randomly divided into two groups ( $n=7$ per group): an OVX group that underwent bilateral ovariectomy to create an OVX model with low estrogen levels and a sham group in which only the periovarian fatty tissue was exteriorized. After ovariectomy, the animals were returned to their cages and fed according to the aforementioned feeding protocol. Before conducting the experiments, ethical approval of this study (SYXK[Yue]2008-0002) was obtained from the Animal Experimental Ethical Inspection Committee of Guangdong Medical Laboratory Animal Center, China. Experiments on rats were performed in accordance with the Guidelines for the Care of Laboratory Animals of the National Institutes of Health.

All animals, weighting 309.9 to $423.3 \mathrm{~g}$, were euthanized with an overdose of sodium pentobarbital (P3761, Sigma, USA) 3 weeks after ovariectomy. The left tibias were excised, harvested, and stored at $-20^{\circ} \mathrm{C}$ until the ultrasound examination.

2.2. Ultrasound Examinations. Before ultrasound scanning, the tibial sample to be measured was thawed in a saline solution for 2 hours. Then, the specimen was vertically fixed with a clamp and immersed in saline in the container. A square region of $0.3 \mathrm{~mm} \times 0.3 \mathrm{~mm}$ on the top surface of

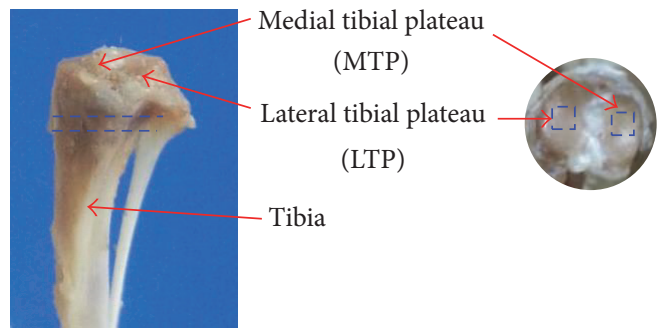

FIGURE 1: Schematic of the areas scanned by ultrasound biomicroscopy (two blue dotted squares $0.3 \mathrm{~mm} \times 0.3 \mathrm{~mm}$ on the medial and lateral tibial plateaus) and micro-CT (two blue dotted lines presenting the volume of interest of the tibial metaphysis, approximately $0.1 \mathrm{~mm}$ in thickness).

cartilage tissue from the medial tibial plateau (MTP) and lateral tibial plateau (LTP) was, respectively, selected as region of interest (ROI) (Figure 1) and perpendicularly placed to the ultrasound beam.

The ultrasound system was comprised of an ultrasound pulser/receiver (Olympus 5900 PR, Panametrics-NDT, Waltham, MA, USA), a $50 \mathrm{MHz}$ transducer (Olympus, PI502-R0.75, Panametrics-NDT), and a computer with a 12-bit A/ D acquisition card (CompuScope 12400, Gage, ON, Canada). The ultrasound scanning over ROI was controlled by customdeveloped software to move the transducer with a 3D translating stage (ETSN400, Tian-Rui-Zhong-Hai Instrument, Beijing, China). After the transducer was properly positioned approximately $1 \mathrm{~cm}$ above the cartilage tissue, the ultrasound examination started. The ultrasound radiofrequency (RF) signals were saved for offline parameter extraction.

Four parameters were then automatically extracted from ultrasound RF signals processed using a self-developed MATLAB program (The MathWorks, Natick, MA, USA): ultrasound roughness index (URI) of the cartilage surface, reflection coefficient of the cartilage surface $\left(\mathrm{RC}_{1}\right)$, reflection coefficient of the cartilage-subchondral bone interface $\left(\mathrm{RC}_{2}\right)$, and the thickness $(h)$ of the cartilage tissue. These parameters were defined by (1)-(4), respectively.

$$
\begin{aligned}
\mathrm{URI} & =\sqrt{\frac{1}{m} \sum_{i=1}^{m}\left(d_{i}-\bar{d}\right)^{2}} \\
\mathrm{RC}_{1} & =\frac{1}{m} \sum_{i=1}^{m} \frac{A_{i}}{A_{\text {ref }}} \times 100 \% \\
\mathrm{RC}_{2} & =\frac{1}{m} \sum_{j=1}^{m} \frac{A_{j}}{A_{\text {ref }}} \times 100 \% \\
h & =\frac{1}{m} \sum_{i=1}^{m}\left(C_{\text {cartilage }} \times \frac{\mathrm{TOF}_{i}}{2}\right) .
\end{aligned}
$$


In these equations, $m$ is the total number of the sampling lines that is equal to 100 in this study. In (1), $d_{i}$ represents the distance from the transducer surface to the cartilage surface in the sampling line $i$ and $\bar{d}$ is the mean of $d_{i}$. In (2) and (3), $A_{i}, A_{j}$, and $A_{\text {ref }}$, respectively, represent the peak-to-peak amplitude of the echoes from the cartilage surface in the sampling line $i$, from the cartilage-bone interface in sampling line $j$, and from a perfect reflector. In (4), $\mathrm{TOF}_{i}$ is the time-offlight from the cartilage surface to the cartilage-bone interface in the sampling line $i$, and $C_{\text {cartilage }}$ is the average sound speed (equal to $1675 \mathrm{~m} / \mathrm{s}$ ) in the cartilage.

2.3. Micro-CT Measurements. After ultrasound examinations, the tibial samples were fixed in $4 \%$ paraformaldehyde solution for more than 72 hours. Then, 3D microarchitecture of the cancellous bone was assessed using a micro-CT system ( $\mu$ CT80, Scanco Medical, AG, Switzerland) with energy settings of $55 \mathrm{kV}$ and $145 \mu \mathrm{A}$. The proximal tibial metaphysis was scanned starting at approximately $1 \mathrm{~mm}$ distal to the growth plate (Figure 1). A total of 100 consecutive tomographic CT slices were taken from proximal to distal with thickness of $0.01 \mathrm{~mm}$ per slice.

In order to analyze the cancellous part of the tibial metaphysis, the trabecular area was contoured manually slice by slice. Then, the trabecular volume of interest (VOI) of the tibial metaphysis was reconstructed by a reconstruction software (Scanco Medical, AG, Switzerland). The micro-CT analysis was performed, giving an isotropic voxel size of $12 \mu \mathrm{m}$. Trabecular number (Tb.N), trabecular thickness (Tb.Th), trabecular separation/spacing (Tb.Sp), connectivity density (Conn.D.), and structure model index (SMI) were extracted to characterize the trabecular microarchitecture of the cancellous bone in menopausal rats. Moreover, BV/TV, which stands for mineralized bone volume over total volume of the given VOI, was used to evaluate relative changes in bone volume density.

2.4. Statistical Analysis. Statistical analyses were performed with SPSS Statistics (Version 20, IBM, Armonk, NY, USA). All values in the text were presented as mean \pm standard deviation (SD). The statistical differences in the acoustic parameters and the micro-CT parameters between the OVX and sham groups were analyzed using Mann-Whitney $U$ test. Significant differences were accepted with $p \leq 0.05$.

\section{Results}

3.1. Results of Ultrasound Examinations. From the results of ultrasound examination of the articular cartilage at MTP (Figure 2), we found significant alterations in the surface cartilage tissue in the OVX group. In comparison with the sham group, $\mathrm{RC}_{1}$ of the OVX group significantly decreased $(p<0.01)$. URI of the OVX group significantly increased $(p<0.05)$ indicating that the surface of articular cartilage became rougher in the early-stage of low estrogen levels. Furthermore, the cartilage thickness in the OVX rats significantly decreased $(p<0.05)$. However, no significant alteration $(p>$
0.05) was found in $\mathrm{RC}_{2}$, which is related to the property of the cartilage-subchondral bone interface.

Similar to the findings of MTP, significant alterations in the surface cartilage tissue at LTP were observed in the OVX group compared with the sham group (Figure 3 ). The significant changes in URI $(p=0.05)$ and cartilage thickness $(p<0.01)$ were observed in the OVX group. However, no significant changes $(p>0.05)$ in $\mathrm{RC}_{1}$ and $\mathrm{RC}_{2}$ were found in the OVX group compared with the sham group.

3.2. Results of Micro-CT Scanning. The 3D reconstruction of the trabecular bone structures in the proximal tibial metaphysis by micro-CT is shown in Figures 4(a) and 4(b). The remarkable change of cancellous bone was not observed between the sham and OVX rats, although some of the connecting rods were missed in the OVX group.

The results of quantitative micro-CT assessment indicate no statistical difference $(p>0.05)$ in bone microarchitectural parameters between the OVX and sham groups (Figures $4(\mathrm{c})-4(\mathrm{~h}))$.

\section{Discussion}

This study focused on the knee joint, especially the tibia, and applied micro-CT and quantitative ultrasound to evaluate alterations in the bone and articular cartilage in an OVX rat model. The primary finding of this study was that significant changes in acoustic parameters of articular cartilage were detected in the rats with ovariectomy, whereas there were insignificant changes in microarchitecture of the cancellous bone. These results indicated that although both articular cartilage and bone are target tissues of estrogens, the degradation of the articular cartilage induced by estrogen deficiency occurred earlier and its characterizations could be detected by quantitative ultrasound. This study provides evidence to previous study suggesting that cartilage degradation might occur in an early stage of low estrogen levels [24].

The cartilage results of this study are similar to those of previous study showing that disuse-induced significant changes in the cartilage tissue [25]. However, disuse simultaneously induced cartilage degradation and bone loss [26]. This may be due to the fact that the articular cartilage and bone are subjected to high loads under gravitation force during daily walking, running, and jumping. Loading is important for keeping knees healthy. Therefore, the two tissues are sensitive to disuse or unloading. Although previous studies reported that estrogens affect the synthesis of both articular cartilage and cancellous bone through the receptor activator nuclear factor-kappa B ligand (RANKL) signaling pathway $[27,28]$, the present study suggested a rapid response of the articular cartilage to estrogen deficiency.

The definite relationship between $\mathrm{OP}$ and $\mathrm{OA}$ still expected more efforts. Herrero-Beaumont et al. reported that both high and low BMD conditions might initiate cartilage degradation in OA [14]. In this study, no significant alterations in the cancellous bone in the $3 \mathrm{wk}$ OVX rats were found, whereas cartilage degradation was shown. Furthermore, a tendency of deterioration in the trabecular 


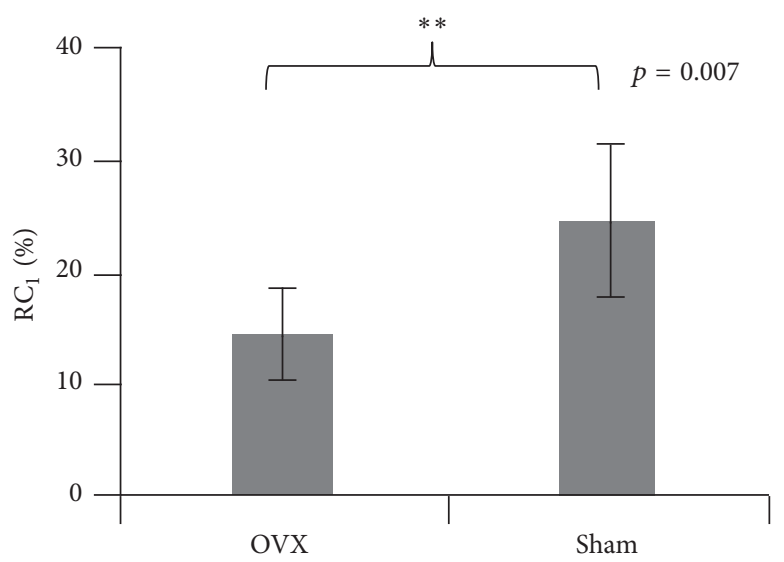

(a)

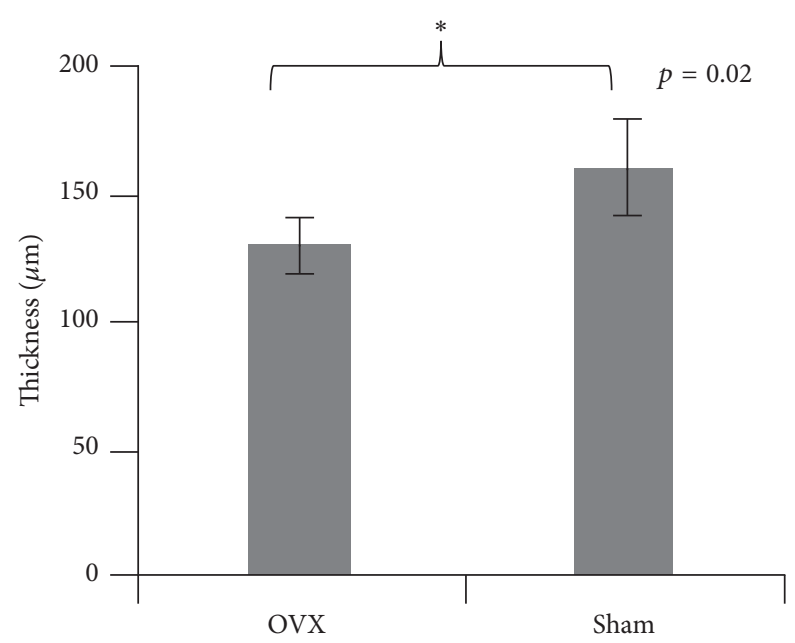

(c)

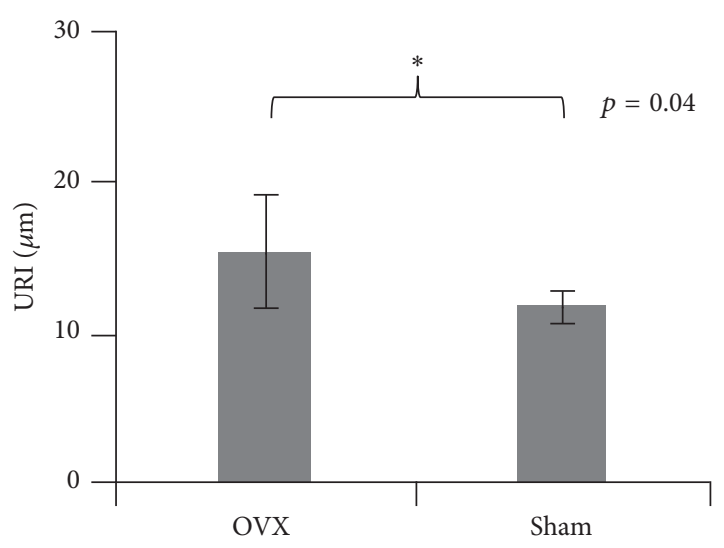

(b)

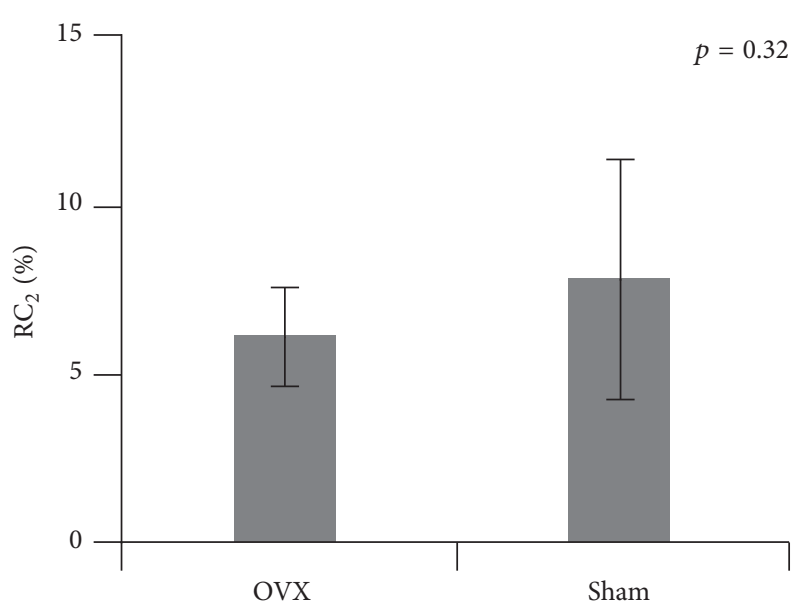

(d)

FIGURE 2: Comparisons of ultrasound results measured at medial tibial plateau (MTP) between the OVX and sham groups. (a) Reflection coefficient $\left(\mathrm{RC}_{1}\right)$ of the cartilage surface; (b) ultrasound roughness index (URI); (c) cartilage thickness $(h)$; and (d) reflection coefficient $\left(\mathrm{RC}_{2}\right)$ of the cartilage-bone interface. ${ }^{*}$ Statistically significant difference at level $p<0.05$ exists between OVX group and sham group. ${ }^{* *}$ Statistically significant difference at level $p<0.01$ exists between OVX group and sham group.

bone was shown in Figure 4, which is consistent with the findings (a decrease in BV/TV and Tb.N with an increase in Tb.Sp and SMI) of previous studies discovered in the $4 \mathrm{wk}$ OVX rats [29], $6 \mathrm{wk}$ OVX rats [30], and $12 \mathrm{wk}$ OVX rats [19]. Brouwers et al. [31], however, showed significant deterioration in BV/TV, Tb.N, and Tb.Th in the $1 \mathrm{wk}$ OVX rats. One possible cause of this divergence might be different ages, weight, and species of the investigated animals. 10-month-old Sprague-Dawley rats used in this study were in an old stage similar to the 50-60-year-old women, whereas 5.5-month rats were young adult rats. The findings of previous studies and this study implicate that the sudden estrogen deficiency after ovariectomy may have dissimilar significant effect on bone structure in the elderly rats to that in adult rats.
The present study has some limitations. Firstly, this study was limited in the detection of OP and OA occurred at the proximal tibia. Because OP and OA may occur at different joints and mechanisms of $\mathrm{OP}$ and $\mathrm{OA}$ are complex, the findings of previous studies are debatable, indicating that a defined relationship between OP and OA has not yet clearly delineated so far. Therefore, this study preliminarily focused on the tibia in the knee joint finding that the early effect of estrogen deficiency on the articular cartilage was faster detected using quantitative ultrasound than that on the cancellous bone detected using micro-CT. Secondly, in consideration of the relatively small sample size of each experimental group, nonparametric analyses were used in this study to reduce some impacts on the statistical results. In future, further studies with more samples are expected on the basis 


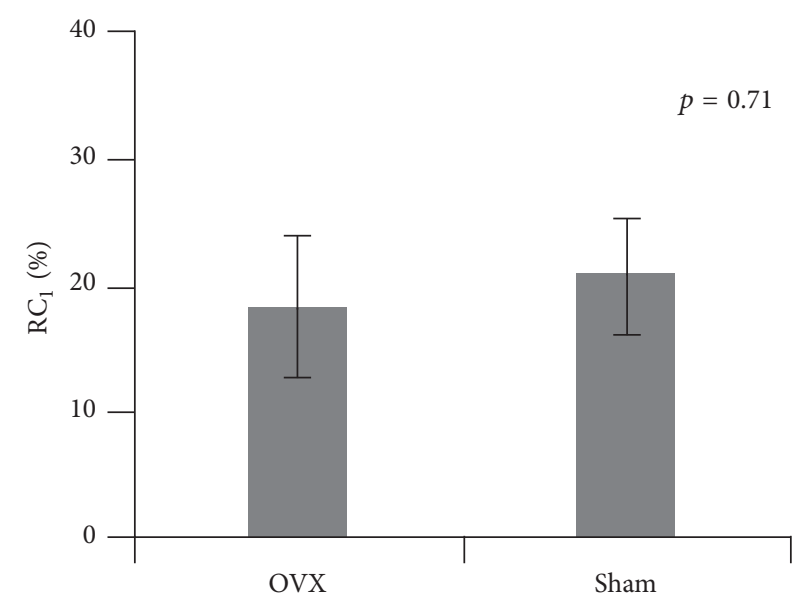

(a)

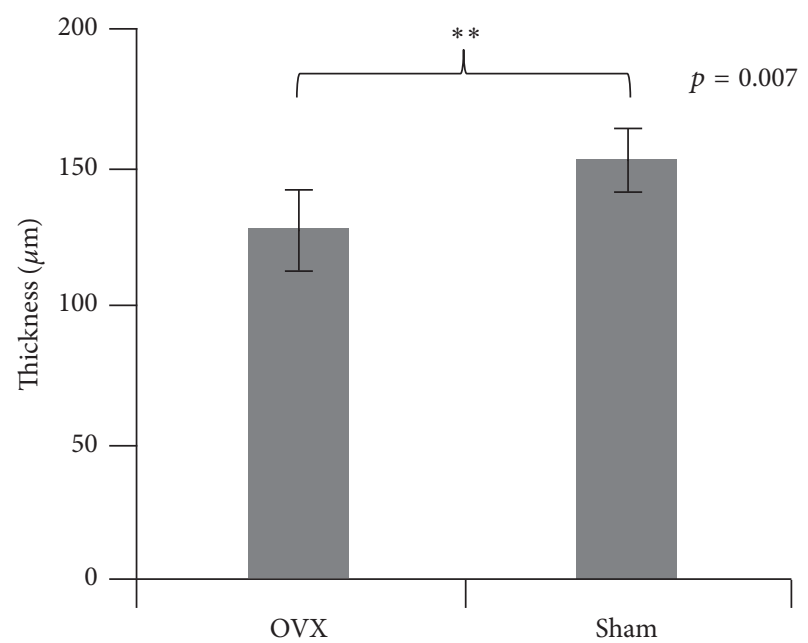

(c)

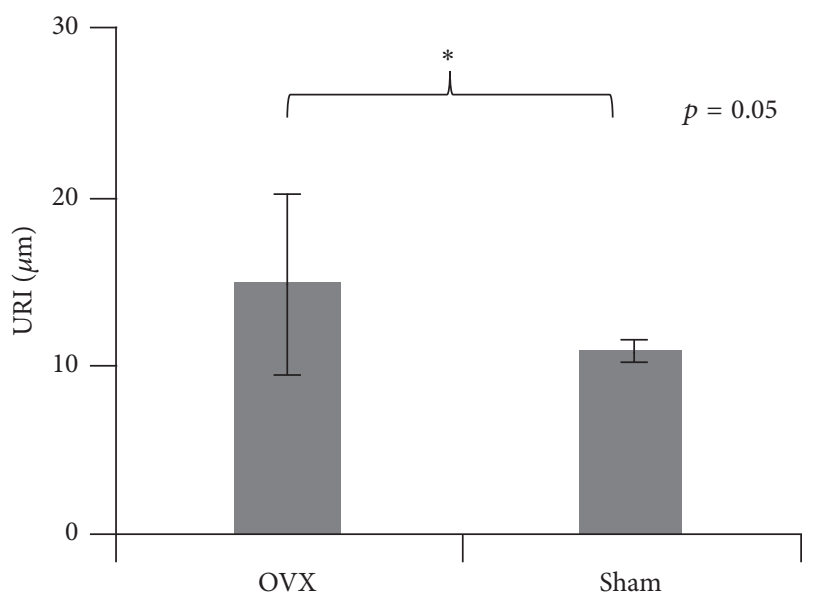

(b)

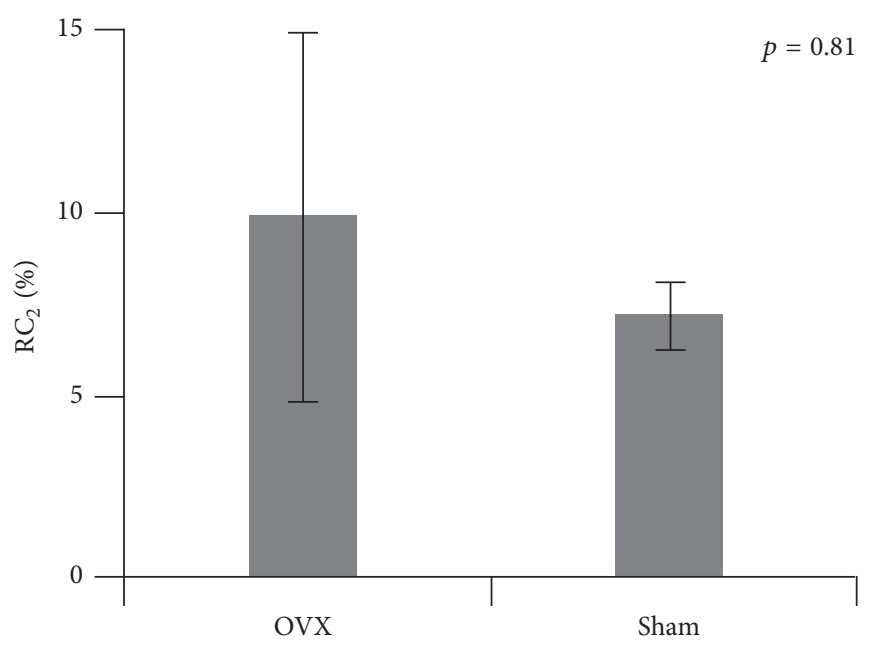

(d)

FIGURE 3: Comparisons of ultrasound results measured at lateral tibial plateau (LTP) between the OVX and sham groups. (a) Reflection coefficient $\left(\mathrm{RC}_{1}\right)$ of the cartilage surface; (b) ultrasound roughness index (URI); (c) cartilage thickness ( $h$ ); and (d) reflection coefficient ( $\left.\mathrm{RC}_{2}\right)$ of the cartilage-bone interface. * Statistically significant difference at level $p<0.05$ exists between OVX group and sham group. ${ }^{* *}$ Statistically significant difference at level $p<0.01$ exists between OVX group and sham group.

of the preliminary result of this study. The other limitation of this study is one time point. In the light of previous studies showing that the bone loss occurred in a relative late-stage (4 weeks after the ovariectomy operation) $[19,29,30]$, this study aimed to detect the early alterations in tibial cartilage and cancellous bone 3 weeks after ovariectomy operation. However, according to our results, changes in the cartilage tissue at more time points such as 1 week or 2 weeks after ovariectomy are needed to be determined in future by quantitative ultrasound during the progression of menopause.

In conclusion, this study demonstrated that degradation of articular cartilage was detected earlier with quantitative ultrasound than that of the cancellous bone in $3 \mathrm{wk}$ OVX rats. These results have implications that deterioration of the cartilage tissue after estrogen deficiency developed faster than cancellous bone. However, due to inconsistent findings of $\mathrm{OP}$ and OA, further studies are expected.

\section{Competing Interests}

The authors have declared that no competing interests exist.

\section{Authors' Contributions}

Qing Wang and Wufan Chen conceived and designed the experiments. Yinong Wang and Zhiwei Liu performed the experiments. Yinong Wang, Zhiwei Liu, and Qing Wang analyzed the data. Qing Wang, Qianjin Feng, and Wufan Chen contributed reagents/materials/analysis tools. Yinong Wang and Qing Wang Contributed to the writing of the manuscript.

\section{Acknowledgments}

This work was supported by National Natural Science Funds of China (no. 81371560), Special Fund for Talent Introduction 


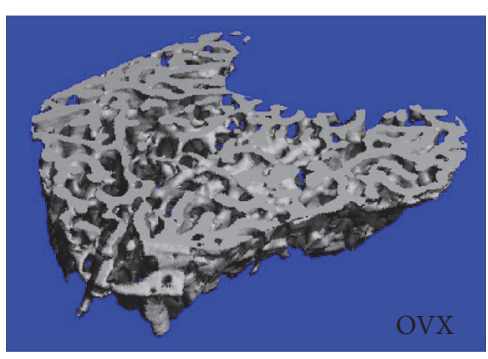

(a)

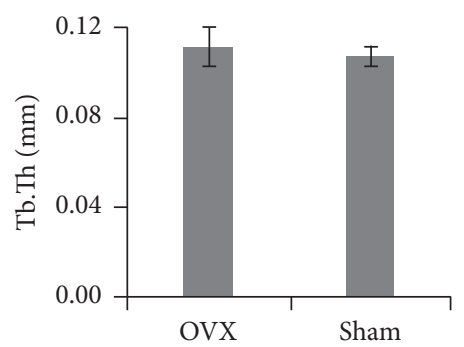

(d)

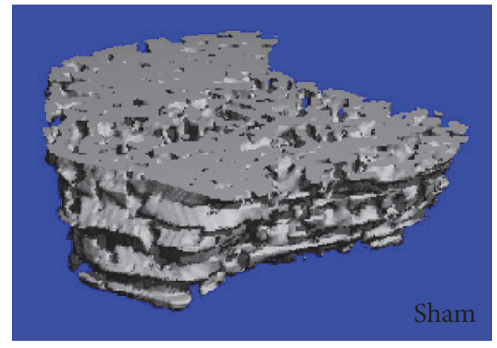

(b)

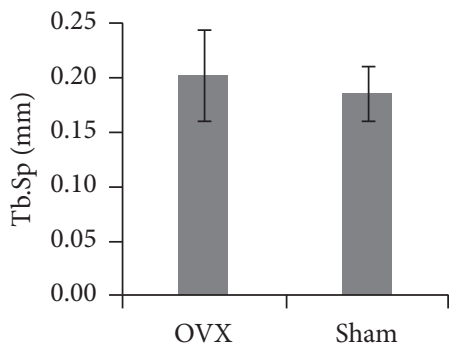

(e)

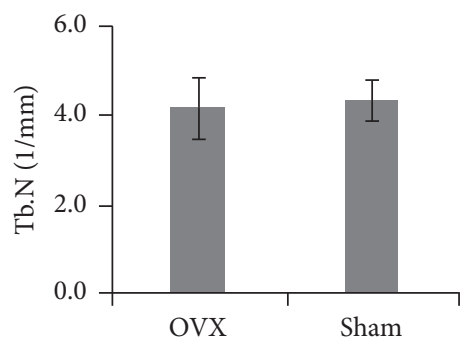

(c)

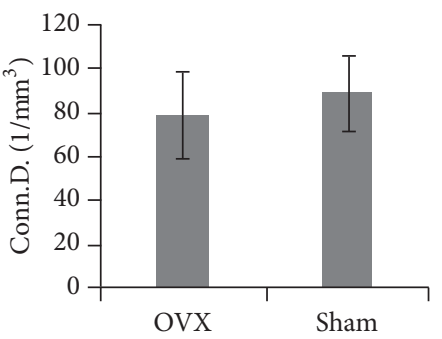

(f)

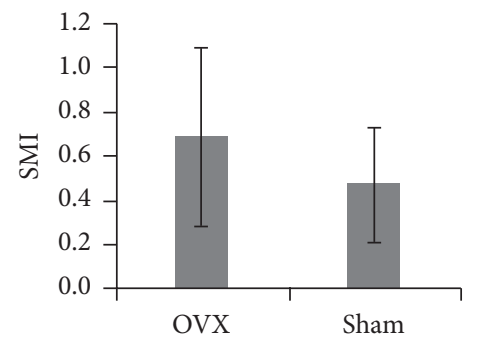

(g)

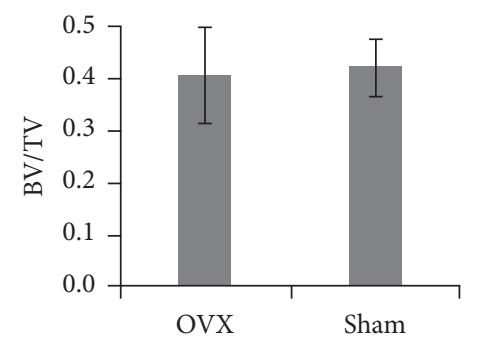

(h)

FIGURE 4: Comparisons of micro-CT results measured at the cancellous bone between the OVX and sham groups. (a) 3D reconstruction of the trabecular bone of the OVX group; (b) 3D reconstruction of the trabecular bone of the sham group: (c) Tb.N; (d) Tb.Th; (e) Tb.Sp; (f) Conn.D.; (g) SMI; (h) BV/TV.

in Guangdong Universities (no. 2050205), Guangdong Provincial Natural Science Funds (no. 2014A030313329), and Guangdong Provincial Technological Funds (no. 2013B021800039).

\section{References}

[1] I. P. Braidman, L. Hainey, G. Batra, P. L. Selby, P. T. K. Saunders, and J. A. Hoyland, "Localization of estrogen receptor $\beta$ protein expression in adult human bone," Journal of Bone and Mineral Research, vol. 16, no. 2, pp. 214-220, 2001.

[2] S. H. Windahl, M. K. Lagerquist, N. Andersson et al., "Identification of target cells for the genomic effects of estrogens in bone," Endocrinology, vol. 148, no. 12, pp. 5688-5695, 2007.

[3] T. Ushiyama, H. Ueyama, K. Inoue, I. Ohkubo, and S. Hukuda, "Expression of genes for estrogen receptors $\alpha$ and $\beta$ in human articular chondrocytes," Osteoarthritis and Cartilage, vol. 7, no. 6, pp. 560-566, 1999.

[4] M. Martin-Millan, M. Almeida, E. Ambrogini et al., "The estrogen receptor-alpha in osteoclasts mediates the protective effects of estrogens on cancellous but not cortical bone," Molecular Endocrinology, vol. 24, no. 2, pp. 323-334, 2010.
[5] K. M. Melville, N. H. Kelly, S. A. Khan et al., "Female mice lacking estrogen receptor-alpha in osteoblasts have compromised bone mass and strength," Journal of Bone and Mineral Research, vol. 29, no. 2, pp. 370-379, 2014.

[6] S. Khosla, M. J. Oursler, and D. G. Monroe, "Estrogen and the skeleton," Trends in Endocrinology and Metabolism, vol. 23, no. 11, pp. 576-581, 2012.

[7] F. S. Hanna, A. J. Teichtahl, A. E. Wluka et al., "Women have increased rates of cartilage loss and progression of cartilage defects at the knee than men: a gender study of adults without clinical knee osteoarthritis," Menopause, vol. 16, no. 4, pp. 666670, 2009.

[8] P. Høegh-Andersen, L. B. Tankó, T. L. Andersen et al., "Ovariectomized rats as a model of postmenopausal osteoarthritis: validation and application," Arthritis research \& therapy, vol. 6, no. 2, pp. R169-R180, 2004.

[9] D. Avci and G. A. Bachmann, "Osteoarthritis and osteoporosis in postmenopausal women: clinical similarities and differences," Menopause, vol. 11, no. 6, part 1, pp. 615-621, 2004.

[10] L. Ichchou, F. Allali, S. Rostom et al., "Relationship between spine osteoarthritis, bone mineral density and bone turn over markers in post menopausal women," BMC Women's Health, vol. 10, article no. $25,2010$. 
[11] J. Multanen, A. Heinonen, A. Häkkinen et al., "Bone and cartilage characteristics in postmenopausal women with mild knee radiographic osteoarthritis and those without radiographic osteoarthritis," Journal of Musculoskeletal Neuronal Interactions, vol. 15, no. 1, pp. 69-77, 2015.

[12] E. Calvo, S. Castañeda, R. Largo, M. E. Fernández-Valle, F. Rodríguez-Salvanés, and G. Herrero-Beaumont, "Osteoporosis increases the severity of cartilage damage in an experimental model of osteoarthritis in rabbits," Osteoarthritis and Cartilage, vol. 15, no. 1, pp. 69-77, 2007.

[13] J. Y. Lee, W. F. Harvey, L. L. Price, J. K. Paulus, B. DawsonHughes, and T. E. McAlindon, "Relationship of bone mineral density to progression of knee osteoarthritis," Arthritis and Rheumatism, vol. 65, no. 6, pp. 1541-1546, 2013.

[14] G. Herrero-Beaumont, J. A. Roman-Blas, R. Largo, F. Berenbaum, and S. Castañeda, "Bone mineral density and joint cartilage: four clinical settings of a complex relationship in osteoarthritis," Annals of the Rheumatic Diseases, vol. 70, no. 9, pp. 1523-1525, 2011.

[15] M. C. Nevitt, N. E. Lane, J. C. Scott et al., "Radiographic osteoarthritis of the hip and bone mineral density," Arthritis \& Rheumatism, vol. 38, no. 7, pp. 907-916, 1995.

[16] K. Okano, M. Ito, K. Aoyagi, M. Osaki, H. Enomoto, and K. Yamaguchi, "Discrepancy in bone mineral densities at different skeletal sites in hip osteoarthritis patients," Modern Rheumatology, vol. 24, no. 2, pp. 340-342, 2014.

[17] M. Lethbridge-Çejku, J. D. Tobin, W. W. Scott Jr. et al., "Axial and hip bone mineral density and radiographic changes of osteoarthritis of the knee: data from the Baltimore Longitudinal Study of Aging," Journal of Rheumatology, vol. 23, no. 11, pp. 1943-1947, 1996.

[18] D. Lim, C.-Y. Ko, D. H. Seo et al., "Low-intensity ultrasound stimulation prevents osteoporotic bone loss in young adult ovariectomized mice," Journal of Orthopaedic Research, vol. 29, no. 1, pp. 116-125, 2011.

[19] F. Li, X. Yang, Y. Yang et al., "Antiosteoporotic activity of echinacoside in ovariectomized rats," Phytomedicine, vol. 20, no. 6, pp. 549-557, 2013.

[20] N. Ibrahim, M. F. Khamis, M. F. M. Yunoh, S. Abdullah, N. Mohamed, and A. N. Shuid, "Targeted delivery of lovastatin and tocotrienol to fracture site promotes fracture healing in osteoporosis model: micro-computed tomography and biomechanical evaluation," PLoS ONE, vol. 9, no. 12, Article ID el15595, 2014.

[21] Q. Wang, Z. Liu, Y. Wang et al., "Quantitative ultrasound assessment of cartilage degeneration in ovariectomized rats with low estrogen levels," Ultrasound in Medicine and Biology, vol. 42, no. 1, pp. 290-298, 2016.

[22] S.-Z. Wang, Y.-P. Huang, S. Saarakkala, and Y.-P. Zheng, "Quantitative assessment of articular cartilage with morphologic, acoustic and mechanical properties obtained using highfrequency ultrasound," Ultrasound in Medicine and Biology, vol. 36, no. 3, pp. 512-527, 2010.

[23] S. Saarakkala, M. S. Laasanen, J. S. Jurvelin, and J. Töyräs, "Quantitative ultrasound imaging detects degenerative changes in articular cartilage surface and subchondral bone," Physics in Medicine and Biology, vol. 51, no. 20, article 017, pp. 5333-5346, 2006.

[24] U. Mouritzen, S. Christgau, H.-J. Lehmann, L. B. Tankó, and C. Christiansen, "Cartilage turnover assessed with a newly developed assay measuring collagen type II degradation products: influence of age, sex, menopause, hormone replacement therapy, and body mass index," Annals of the Rheumatic Diseases, vol. 62, no. 4, pp. 332-336, 2003.

[25] Q. Wang, X. Guo, M.-Q. Liu, X.-Y. Wang, and Y.-P. Zheng, "Effect of laser acupuncture on disuse osteoarthritis: an ultrasound biomicroscopic study of patellar articular cartilage in rats," Evidence-based Complementary and Alternative Medicine, vol. 2012, Article ID 838420, 8 pages, 2012.

[26] Q. Wang, X. Guo, X. Y. Wang et al., "Effect of laser acupuncture therapy on bone and articular cartilage under simulated microgravity," in 6th World Congress of Biomechanics (WCB 2010). August 1-6, 2010 Singapore: In Conjunction with 14th International Conference on Biomedical Engineering (ICBME) and 5th Asia Pacific Conference on Biomechanics (APBiomech), vol. 31 of IFMBE Proceedings, pp. 852-855, Springer, Berlin, Germany, 2010.

[27] J. Kular, J. Tickner, S. M. Chim, and J. Xu, "An overview of the regulation of bone remodelling at the cellular level," Clinical Biochemistry, vol. 45, no. 12, pp. 863-873, 2012.

[28] J. Xu, H. F. Wu, E. S. M. Ang et al., "NF- $\kappa$ B modulators in osteolytic bone diseases," Cytokine and Growth Factor Reviews, vol. 20, no. 1, pp. 7-17, 2009.

[29] M. Ito, A. Nishida, T. Nakamura, M. Uetani, and K. Hayashi, "Differences of three-dimensional trabecular microstructure in osteopenic rat models caused by ovariectomy and neurectomy," Bone, vol. 30, no. 4, pp. 594-598, 2002.

[30] J. Yang, S. M. Pham, and D. L. Crabbe, "High-resolution Micro-CT evaluation of mid- to long-term effects of estrogen deficiency on rat trabecular bone," Academic Radiology, vol. 10, no. 10, pp. 1153-1158, 2003.

[31] J. E. M. Brouwers, F. M. Lambers, B. Van Rietbergen, K. Ito, and R. Huiskes, "Comparison of bone loss induced by ovariectomy and neurectomy in rats analyzed by in vivo micro-CT," Journal of Orthopaedic Research, vol. 27, no. 11, pp. 1521-1527, 2009. 


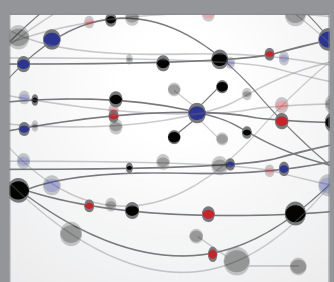

The Scientific World Journal
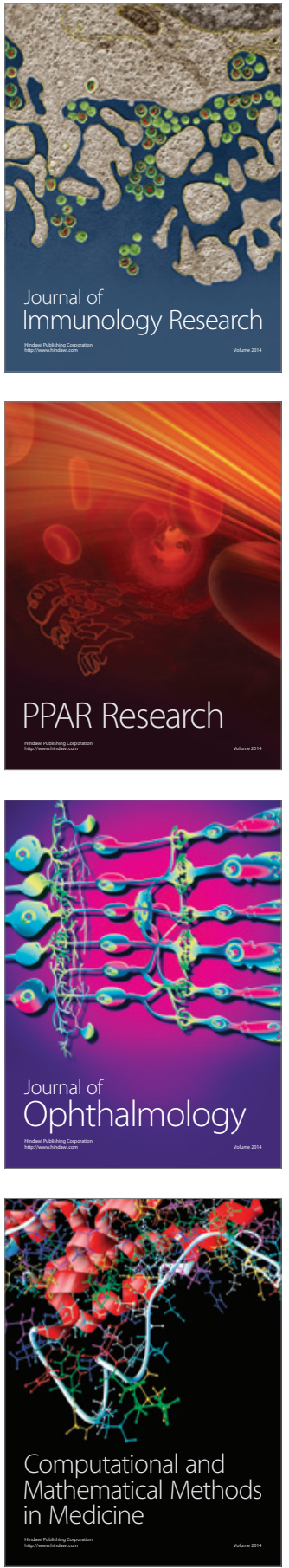

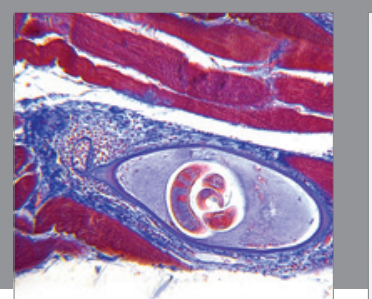

Gastroenterology Research and Practice
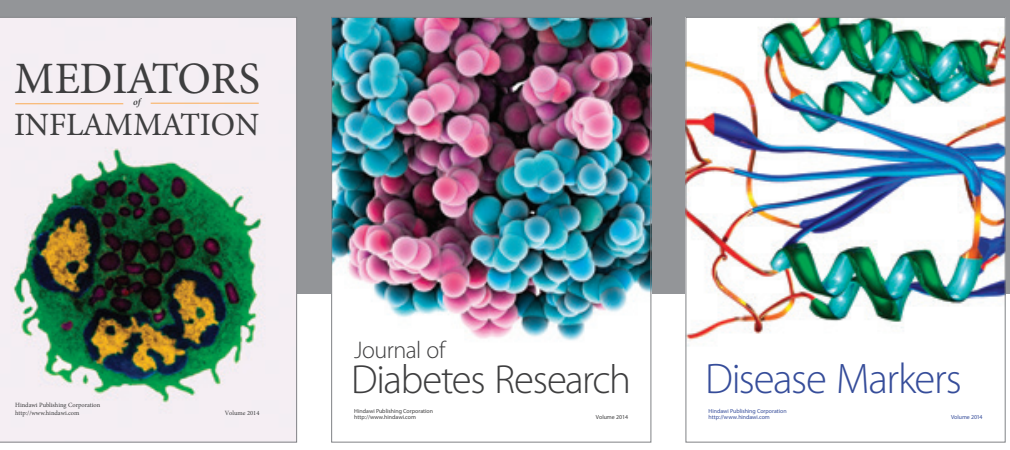

Disease Markers

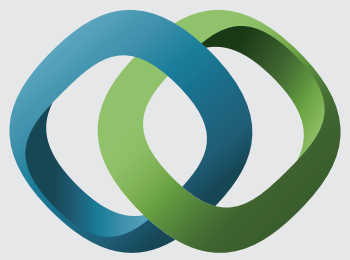

\section{Hindawi}

Submit your manuscripts at

https://www.hindawi.com
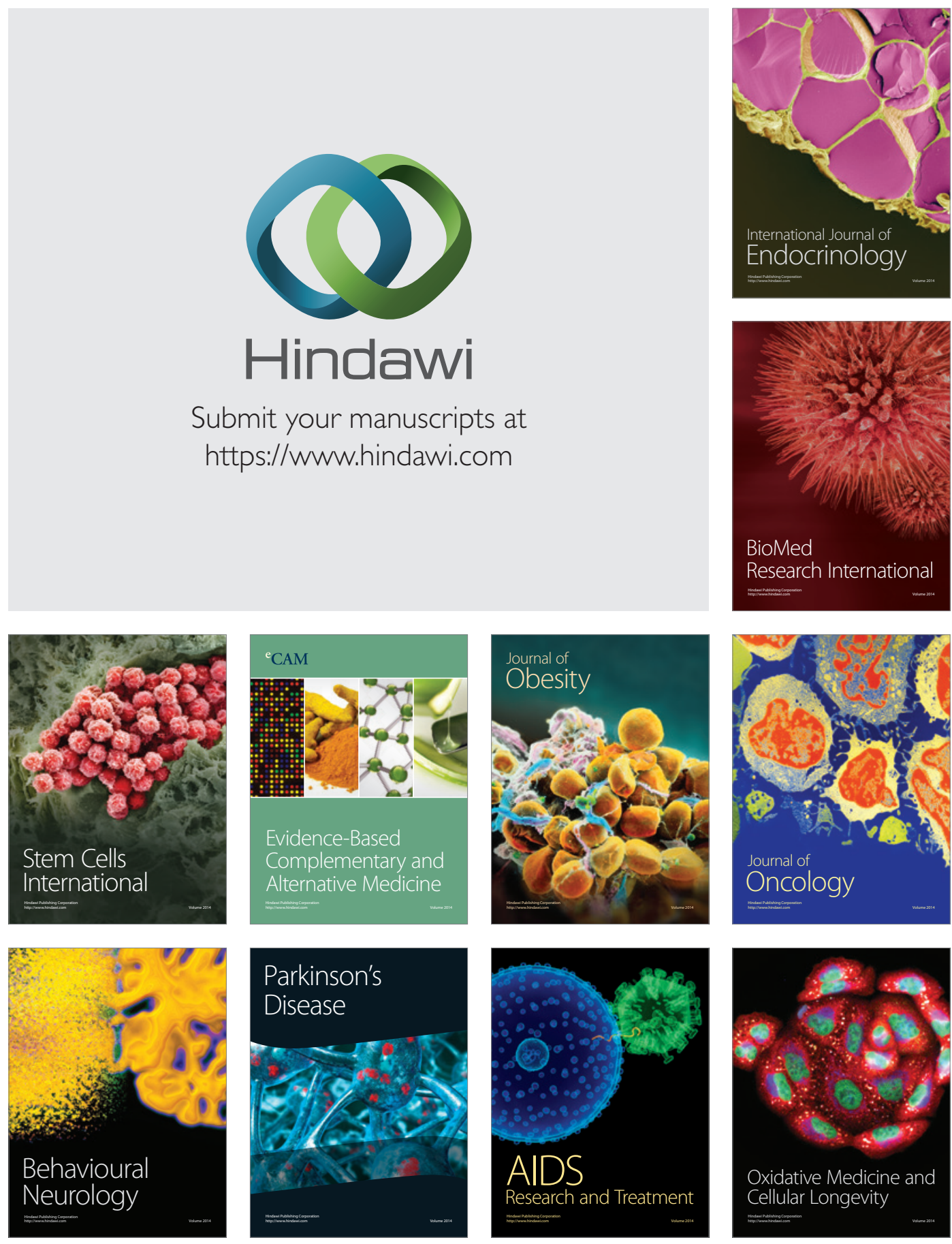